\title{
APPLICATIONS OF THE KKM-PRINCIPLE TO PROLLA TYPE THEOREMS
}

\author{
S. SESSA AND S. P. SINGH
}

\begin{abstract}
We prove some theroems of Prolla type [13] using a well known KKM-principle of Ky Fan [6], so generalizing several results known in the literature.
\end{abstract}

The following theorem due to Prolla [13] was proved using tools from approximation theory and the Bohnenblust and Karlin theorem [2]:

Theorem 1. Let $X$ be a nonempty compact convex subset of a normed linear space $E$ and $g: X \rightarrow X$ be a continuous almost affine onto map. Then for each continuous map $f: X \rightarrow E$, there exists a point $x_{0} \in X$ such that

$$
\left\|g x_{0}-f x_{0}\right\|=\inf \left\{\left\|x-f x_{0}\right\|: x \in X\right\} .
$$

Let $X$ be nonempty convex subset of a normed linear space $E$ and $g: X \rightarrow$ $E$. We recall that $g$ is almost affine on $X$ if

$$
\left.\| g\left(\lambda x_{1}\right)+(1-\lambda) x_{2}\right)-y\|\leq \lambda \cdot\| g x_{1}-y\|+(1-\lambda) \cdot\| g x_{2}-y \|
$$

for all $x_{1}, x_{2} \in X, \lambda \in[0,1]$ and $y \in E$.

The following result, due to Ky Fan [6], extends known results on the KKMprinciple.

Theorem 2. Let $Y$ be a nonempty convex subset of a Hausdorff topological vector space $E$ and $X$ be a nonempty subset of $Y$. For each $x \in X$, let $F x$ be a

Received May 1, 1990. 
relatively closed subset of $Y$ such that $F$ is a KKM-map. If there is a nonempty subset $X_{0}$ of $X$ such that the intersection $B=\cap_{x \in X_{0}} F x$ is compact and $X_{0}$ is contained in a compact convex subset $S$ of $Y$, then $\cap_{x \in X} F x \neq \phi$.

Remark 1. As noted by Lin [11], the set $B$ is necessarily nonempty.

A very good source of reference on KKM-Principle is due to Granas [7] where several applications are given. The following result due to Allen [1] follows from Theorem 2 as a Corollary.

Theorem 3. Let $X$ be a nonempty convex subset of a Hausdorff topological vector space. Let $\Phi: X \times X \rightarrow R$ be a real valued function satisfying the following properties:

(i) For each fixed $x \in X, \Phi(x, y)$ is a lower semicontinuous function of $y$ on $X$,

(ii) For each fixed $y \in X, \Phi(x, y)$ is a quasiconcave function of $x$ on $X$,

(iii) $\Phi(x, x) \leq 0$ for all $x \in X$,

(iv) $X$ has a nonempty compact convex subset $X_{0}$ such that the set $B=\{y \in$ $X: \Phi(x, y) \leq 0$ for all $\left.x \in X_{0}\right\}$ is compact.

Then there exists some $y_{0} \in X$ such that $\Phi\left(x, y_{0}\right) \leq 0$ for all $x \in X$.

We recall that a real function $f$ on a convex set $X$ is quasiconcave if the $\{x \in X: f(x)>t\}$ is convex for all $t \in R$ and it is lower semicontinuous if the set $\{x \in X: f(x) \leq t\}$ is closed in $X$ for all $t \in R$.

Remark 2. As noted by Shih and Tan [14], the coercive condition (iv) is a unification of the two coercive conditions given by Allen [1] and Brezis, Nirenberg and Stampacchia [3]. It is easily seen that (ii) and (iii) imply that $B$ is nonempty.

We prove the following:

Theorem 4. Let $X$ be a nonempty compact convex of a normed linear space $E$ and let $g: X \rightarrow E$ be a continuous map such that

(a) $g(X)$ is convex and $g^{-1}(y)$ is convex for every $y \in g(X)$. 
Then for each continuous map $f: X \rightarrow E$, either there exists some $x_{0} \in X$ such that $g x_{0}=f x_{0}$ or for any $y \in g(X)$ :

$$
0<\left\|g x_{0}-f x_{0}\right\| \leq\left\|y-f x_{0}\right\| .
$$

Remark 3. We note that the almost affine map $g: X \rightarrow X$ of Theorem 1 satisfies condition (a). Indeed, let $y \in g(X)=X$ and $x_{1}, x_{2} \in g^{-1}(y)$. Then we have for each $\lambda \in[0,1]$ :

$$
\left\|g\left(\lambda x_{1}+(1-\lambda) x_{2}\right)-y\right\| \leq \lambda \cdot\left\|g x_{1}-y\right\|+(1-\lambda) \cdot\left\|g x_{2}-y\right\|=0,
$$

i.e. $\lambda x_{1}+(1-\lambda) x_{2} \in g^{-1}(y)$. Hence $g^{-1}(y)$ is convex for any $y \in g(X)$. This implies that Theorem 1 follows from Theorem 4.

We need the following theorem, which was established by Komiya [10] combining Lemma, 1 of $\mathrm{Ha}$ [8] and Prop. 2 of Browder [4]:

Theorem 5. Let $A$ be a nonempty convex subset of a Hausdorff linear topological space $E$ and let $B$ be a nonempty compact convex subset of a Hausdorff linear topological space $F$. Let $S: A \rightarrow 2^{B}$ be an upper semicontinuous set-valued mapping such that $S(a)$ is a nonempty closed convex subset of $B$ for each $a \in A$ and $T: B \rightarrow 2^{A}$ be a set-valued mapping such that $T(b)$ is a nonempty convex subset of $A$ and $T^{-1}(a)=\{b \in B: a \in T(b)\}$ is open in $B$ for each $a \in A$. Then there exist a point $a_{0} \in A$ and a point $b_{0} \in B$ such that $a_{0} \in T\left(b_{0}\right)$ and $b_{0} \in S\left(a_{0}\right)$.

We recall that a set-valued mapping $S: A \rightarrow 2^{B}$ is upper semicontinuous if $S^{-1}(C)=\{a \in A: S(a) \cap C \neq \phi\}$ is closed in $A$ for every closed subset $C$ of $B$.

Proof of theorem 4. Assuming the negation of the second alternative, we have (b) for each $x \in X$ such that $\|g x-f x\|>0$, there exists a point $y \in g(X)$ such that

$$
\|g x-f x\|>\|y-f x\| .
$$

Let $g x \neq f x$ for any $x \in X$. Define a set-valued mapping $T: X \rightarrow 2^{g(X)}$ by setting $T(x)=\{y \in g(X):\|g x-f x\|>\|y-f x\|\}$ for any $x \in X$. 
Since $g(X)$ is convex and (b) holds, $T(x)$ is a nonempty convex subset of $g(X)$. Further, $X \backslash T^{-1}(y)=\{x \in X:\|g x-f x\| \leq\|y-f x\|\}$ is closed in $X$ since $f$ and $g$ are continuous, so $T^{-1}(y)$ is open in $X$ for any $y \in g(X)$. Now, define a set-valued mapping $S: g(X) \rightarrow 2^{X}$ by setting $S(y)=g^{-1}(y)$ for each $y \in g(X)$. Since $g$ is closed and $S^{-1}(C)=g(C)$ for any closed subset $C$ of $X$, we have that $S$ is an upper semicontinuous mapping such that, by (a), $S(y)$ is a nonempty closed convex subset of $X$ for each $y \in g(X)$. Using Theorem 5 with $A=g(X)$ and $B=X$, there exists two points $y_{0} \in g(X)$ and $x_{0} \in X$ such that $y_{0} \in T x_{0}$ and $x_{0} \in g^{-1}\left(y_{0}\right)$. This implies that $\left\|g x_{0}-f x_{0}\right\|>\left\|y_{0}-f x_{0}\right\|=\left\|g x_{0}-f x_{0}\right\|$, a contradiction.

Remark 4. It is clear that the above proof can be adopted to prove the more general Theorem 3 of $\mathrm{Ha}$ [9], of which Theorem 4 is a special case.

Now we prove some results where the compactness on the referential set $X$ is relaxed. In the sequel, $w$ stands for the weak topology.

Theorem 6. Let $X$ be a nonempty convex subset of a normed linear space $E$. Let $f:(X, w) \rightarrow(E,\|\|)$ be a sequentially strongly continuous map and $g:(X, w) \rightarrow(E, w)$ be a sequentially weakly continuous map such that $\left(a_{1}\right) g^{-1}([y, z])$ is convex for $y, z \in g(X)$.

$\left(b_{1}\right)$ Moreover, let $X_{0}$ be a nonempty weakly compact convex subset of $X$ such that the set $B=\left\{y \in X:\left\|g y-\int y\right\| \leq\|g x-f y\|\right.$ for all $\left.x \in X_{0}\right\}$ is weakly compact. Then there exists a point $x_{0} \in B$ such that

$$
\left\|g x_{0}-f x_{0}\right\|=\inf \left\{\left\|g x-f x_{0}\right\|: x \in X\right\} .
$$

If $g(X)=X$, then (1) is satisfied.

Proof. Define a set-valued mapping $F: X \rightarrow 2^{X}$ by setting $F(x)=\{y \in$ $X:\|g y-f y\| \leq\|g x-f y\|\}$ for each $x \in X . F x$ is weakly closed. Indeed, let $\left\{y_{\alpha}\right\}$ be in $F x$ converging weakly to $y$. Then $g y_{\alpha}-f y_{\alpha} \rightarrow g y-f y$ weakly and $g x-f y_{\alpha} \rightarrow g x-f y$ strongly. Now,

$$
\|g y-f y\| \leq \liminf \operatorname{in}_{\alpha}\left\|g y_{\alpha}-f y_{\alpha}\right\| \leq \liminf \inf _{\alpha}\left\|g x-f y_{\alpha}\right\|=\|g x-f y\|,
$$


i.e. $y \in F x$. We show that $F$ is a KKM-map, i.e. the convex hull co $\left\{x_{1}, x_{2}, \ldots\right.$, $\left.x_{n}\right\}$ of every finite subset $\left\{x_{1}, x_{2}, \ldots, x_{n}\right\}$ of $X$ is contained in the corresponding union $\bigcup_{i=1}^{n} F x_{i}$. Indeed, let $z \in \operatorname{co}\left\{x_{1}, \ldots, x_{n}\right\}$ and assume that $z \notin F x_{i}$ for any $i=1, \ldots, n$. Then $x_{i} \notin F^{-1}(z)$, i.e. $x_{i} \in X \backslash F^{-1}(z)=\{x \in X:\|g z-f z\|>$ $\|g x-f z\|\}$ for any $i=1, \ldots, n$. As proved in Theorem 1 of $\operatorname{Lin}[12], X \backslash F^{-1}(z)$ is convex. Indeed, let $z_{1}, z_{2} \in X \backslash F^{-1}(z)$ and let $g z_{1}=u_{1}, g z_{2}=u_{2}$. Since $g^{-1}\left(\left[u_{1}, u_{2}\right]\right)$ is convex by $\left(a_{1}\right)$, we have $\lambda z_{1}+(1-\lambda) z_{2} \in g^{-1}\left(\left[u_{1}, u_{2}\right]\right)$ for $\lambda \epsilon$ $[0,1]$. Thus $g\left(\lambda z_{1}+(1-\lambda) z_{2}\right) \in\left[u_{1}, u_{2}\right]$, i.e. for any $\lambda \in[0,1]$ there exists $h_{\lambda} \in$ $[0,1]$ such that $g\left(\lambda z_{1}+(1-\lambda) z_{2}\right)=h_{\lambda} u_{1}+(1-h \lambda) u_{2}=h_{\lambda} g\left(z_{1}\right)+\left(1-h_{\lambda}\right) g\left(z_{2}\right)$.

Then

$$
\left\|g\left(\lambda z_{1}+(1-\lambda) z_{2}\right)-f z\right\|=h_{\lambda} \cdot\left\|g z_{1}-f z\right\|+\left(1-h_{\lambda}\right) \cdot\left\|g z_{2}-f z\right\|<\|g z-f z\| .
$$

This means that $\lambda z_{1}+(1-\lambda) z_{2} \in X \backslash F^{-1}(z)$ for $\lambda \in[0,1]$, i.e. $X \backslash F^{-1}(z)$ is convex. Then co $\left\{x_{1}, \ldots, x_{n}\right\} \subset X \backslash F^{-1}(z)$, i.e. $z \notin F^{-1}(z)$, a contradiction to the fact $x \in F(x)$, i.e. $x \in F^{-1}(x)$ for each $x \in X$. Since $\left(b_{1}\right)$ holds, all the hypothesis of Theorem 2 (with $X=Y$ and $S=X_{0}$ ) are satisfied in $(E, w)$. Then $\cap_{x \in X} F(x) \neq \phi$ and a point $x_{0}$ of this intersection, contained in $B$, verifies our conclusion.

Remark 5. Condition $\left(a_{1}\right)$ is the same condition $\left(c^{\prime}\right)$ used by Ha [9] and Lin [12].

Corollary 1. Let $X$ be a nonempty weakly compact convex subset of a normed linear space $E$. Let $f, g$ satisfy conditions of Theorem 6 including $\left(a_{1}\right)$. Then there exists a point $x_{0} \in X$ satisfying (2).

Proof. It suffices to observe that condition $\left(b_{1}\right)$ of Theorem 6 is fulfilled in this case by taking any nonempty weakly closed convex subset $X_{0}$ (in particular, $X$ itself) of $X$.

Corollary 2. Let $X$ be a nonempty compact convex subset of a normed linear space $E$, let $f, g: X \rightarrow E$ be continuous and $g$ satisfies condition $\left(a_{1}\right)$. Then there exists a point $x_{0} \in X$ such that (2) holds. 
Proof. In this case, $(X, w) \equiv(X,\|\|)$ since $(X,\|\|)$ is strongly compact and $(X, w)$ is separated. Consequently, the concepts of continuity and sequential continuity coincide since $X$ is metrizable. Thus the thesis follows from Corollary 1.

Remark 6. Corollary 2 is also a corollary of Theorem 3 of Ha [9] (with condition $\left(a_{1}\right)$ ) by taking $E=F$ as normed linear space. Corollary 2 is also a corollary of Theorem 2 of $\operatorname{Lin}$ [12] by assuming $E=F$. Note that Lin [12] derived his Theorem 1 (which is Theorem 3 of $\mathrm{Ha}$ [9] under condition $\left(a_{1}\right)$ ) directly by the famous Lemma 1 of Fan [5] and his Theorem 2 from his Lemma [11], which includes Lemma 1 of Fan [5]. Following the lines of proof of Theorem 6, it is easy to derive Theorem 2 of Lin [12] directly from Theorem 2.

Remark 7. As pointed out by Lin [11], [12], condition $\left(b_{1}\right)$ of Theorem 6 can be replaced by the following condition [6, Theorem 7], [15]:

$\left(b_{1}^{\prime}\right)$ Let $X_{0}$ be a nonempty weakly compact convex subset of $X$ and $K$ be a nonempty weakly compact subset of $X$ such that for every $y \in X \backslash K$, there exists a point $x \in X_{0}$ for which $\|g y-f y\|>\|g x-f y\|$. The conclusion of Theorem 6 will be: there exists a point $x_{0} \in K$ such that (2) holds.

We state and prove the following using Theorem 3.

Theorem 7. Let $X$ be a nonempty convex subset of a normed linear space $E, f:(X, w) \rightarrow(E,\|\|)$ be sequentially strongly continuous and $g:(X, w) \rightarrow$ $(E, w)$ be sequentially weakly continuous and almost affine on $X$. Moreover, condition $\left(b_{1}\right)$ holds. Then there exists a point $x_{0} \in B$ such that (2) holds. If $g(X)=X$, then (1) is satisfied.

Proof. Define $\Phi: X \times X \rightarrow R$ by $\Phi(x, y)=\|g y-f y\|-\|g x-f y\|$ for all $x, y \in X$. For each $x \in X$, then $\Phi(x, y)$ is a weakly lower semicontinuous function of $y$ on $X$ (cfr. proof of Theorem 6). For any $y \in X$ and $t \in R$, let $C_{t}(y)=\{x \in X: \Phi(x, y)>t\}$. We show that this set is convex. If $x_{1}, x_{2} \in C_{t}(y)$ 
and $\lambda \in[0,1]$, we have

$$
\begin{aligned}
& \Phi\left(\lambda x_{1}+(1-\lambda) x_{2}, y\right) \\
= & \|g y-f y\|-\| g\left(\lambda x_{1}+(1-\lambda) x_{2}-f y \|\right. \\
\geq & \|g y-f y\|-\lambda \cdot\left\|g x_{1}-f y\right\|-(1-\lambda) \cdot\left\|g x_{2}-f y\right\| \\
> & \|g y-f y\|+\lambda(t-\|g y-f y\|)+(1-\lambda)(t-\|g y-f y\|) \\
= & t
\end{aligned}
$$

since $g$ is almost affine. All the conditions of Theorem 3 are satisfied and the thesis follows.

Remark 8. Of course, condition $\left(b_{1}\right)$ can be replaced in Theorem 7 by condition $\left(b_{1}^{\prime}\right)$.

Corollary 3. Let $X$ be a nonempty weakly compact convex of a normed linear space $E$. Let $f, g$ be as in Theorem 7 and $g(X)=X$. Then there exists a point $x_{0} \in X$ satisfying (1).

Remark 9. Theorem 1 is clearly a consequence of Corollary 3. If $g$ is the identity function of $X$, Theorem 6 or Theorem 7 give Theorem 3 of Singh, Sehgal and Smithson [15].

Remark 10. It is evident that Theorem 6 and Theorem 7 can be established in the more general context of a locally convex Hausdorff topological vector space $E$. In this case, as observed by $\operatorname{lin}[12]$, conditions $\left(b_{1}\right)$ and $\left(b_{1}^{\prime}\right)$ are replaced respectively by the following:

$\left(c_{1}\right)$ For any continuous seminorm $p$ on $E$, there exists a nonempty weakly compact convex subset $X_{0}(p)$ of $X$ such that the set $B(p)=\{y \in X$ : $p(g y-f y) \leq p(g x-f y)$ for all $\left.x \in X_{0}(p)\right\}$ is weakly compact.

$\left(c_{1}^{\prime}\right)$ For any continuous seminorm $p$ on $E$, there exists a nonempty weakly compact convex subset $X_{0}(p)$ of $X$ and a nonempty weakly compact subset $K(p)$ such that for every $y \in X \backslash K(p)$, there exists a point $x \in X_{0}(p)$ for which $p(g y-f y) \leq p(g x-f y)$. 
Of course, in Theorem 7 one defines that $g$ is almost affine on $X$ for any continuous seminorm $p, \lambda \in[0,1], x_{1}, x_{2} \in X, y \in E$, it is $p\left(g\left(\lambda x_{1}+(1-\lambda) x_{2}\right)-\right.$ $y) \leq \lambda p\left(g x_{1}-y\right)+(1-\lambda) p\left(g x_{2}-y\right)$.

In this case, the proof of Theorem 6 and 7 is deduced via the Hahn-Banach theorem (cfr. proof of Lemma 1 of [15]) and the conclusion will be:

Either there exists a point $x_{0} \in X$ such that $g x_{0}=f x_{0}$ or there exists a continuous seminorm $p$ on $F$ and a point $x_{0} \in B(p)$ (resp. $x_{0} \in K(p)$ ), if $\left(c_{1}\right)$ (resp. $\left.\left(c_{1}^{\prime}\right)\right)$ is assumed, such that $0<p\left(g x_{0}-f x_{0}\right) \leq p\left(y-f x_{0}\right)$ for all $y \in g(X)$.

In this way, of course, for $g=$ identity function on $X$, one deduces Theorem 1 of [15].

\section{Acknowledgement}

Thanks are due to Profs. Lin and Tan for providing with reprints of their papers.

\section{References}

[1] G. Allen, "Variational inequalities, Complementarity problems and duality theorems", $J$. Math. Anal. Appl., 58 (1977) 1-10.

[2] H. F. Bohnenblust and S. Karlin, "On a theorem of Ville, in: Contributions to the Theory of Games", H. W. Kuhn and A. W. Tucker, Eds., Vol. 1, Ann. Math. Studies 24, Princeton Univ. Press (1950), 155-160.

[3] H. Brezis, L. Nirenberg and G. Stampacchia, "A remark on Ky Fan's minimax principle", Boll. Un. Mat. Ital., 6 (1972), 293-300.

[4] F. Browder, "Coincidence theorems, minimax theorems and variational inequalities", Contemporary Math., 26 (1984), 67-80.

[5] K. Fan, "Extensions of two fixed point theorems of F. E. Browder", Math. Z., 112 (1969), 234-240.

[6] K. Fan, "Some properties of convex sets related to fixed point theorems", Math, Ann., 266 (1984), 519-537.

[7] A. Granas, "KKMM-maps and their applications to nonlinear problems", The Scottish Book, Ed. R. D. Mauldin, Birkhauser, (1982), 45-61.

[8] C. W. Ha, "Minimax and fixed point theorems", Math. Ann., 248 (1980), 73-77.

[9] C. W. Ha, "Extensions of two fixed point theorems of Ky Fan", Math. Z., 190 (1985), 13-16.

[10] H. Komiya, "Coincidence theorem and saddle point theorem", Proc. Amer. Math. Soc., 96 (1986), 599-602. 
[11] T. C. Lin, "Convex sets, fixed points, variational and minimax inequalities", Bull. Austral. Math. Soc., 34. (1986), 107-117.

[12] T. C. Lin, "Coincidence and fixed points", to appear.

[13] J. B. Prolla, "Fixed point theorems for set-valued mappings and existence of best approximants", Numer Funct. Anal. and Optimiz., 5 (1982-83), 449-455.

[14] M. H. Shih and K. K. Tan, "Covering theorems of convex sets to fixed point theorems, in: Nonlinear and Convex Analysis (Bor-Luh Lin and S. Simons, Eds.) Lecture Notes in Pure and Appl. Math. 107, Marcel Dekker (1987), 235-244.

[15] V. M. Sehgal, S. P. Singh and R. E. Smithson, "Nearest points and some fixed point theorems for weakly compact sets", J. Math. Anal. Appl., 128 (1987), 108-111.

Università di Napoli, Facoltà di Architettura, Istituto Matematico, Via Monteoliveto, 3, 80134 Napoli, ITALY.

Department of Mathematics and Statistics, Memorial University of Newfoundland, St. John's, NFLD, CANADA AIC 5S7. 